9. Ценообразование и сметное нормирование в строительстве: учебное пособие / М. А. Королева. - 2-е изд., доп. и перераб. - Екатеринбург : Изд-во Урал. ун-та, 2014. - 263с.

\title{
References:
}

1. Method for determining the cost of construction products in the territory of the Russian Federation MDS 81-35.2004. Resolution of the State Construction Committee of Russia from 05.03.2004 № 15/1 with the changes set out in the letter Rosstroy from 23.06.2004 number AP-3230/06 (hereinafter - MDS 8135.2004), registration number 94 of 15.12.2009 federal register estimate standards.

2. On the results of socio-economic development of the Russian Federation in $2015 . \quad$ - [Electronic resource] -Mode http://economy.gov.ru/wps/wcm/connect/bfeda678-29cd-44ae-b07b-d3c6d access.

3. The introduction of the new state estimate standards. Order of the Ministry of Construction and Housing and Communal Services of the Russian Federation № 31 / pr. from 01.30.2014.

4. On some issues of organization and conduct of the state examination of project documentation and engineering survey results in relation to objects, construction of which is financed with funds from the federal budget, as well as on the procedure for determining the validation of the estimated cost of these facilities. RF Government Decree of 23.9.2013 number 840.

5. On the procedure for the validation determination of the estimated cost of capital construction, construction of which is financed with funds from the federal budget. RF Government Resolution of 18.05. 2009 № 427.

6. Approval of the estimated classification standards to be applied in the determination of the estimated cost of capital construction, construction of which is financed with funds from the federal budget. Order of 04.12.2012 number 76 . HS (registered in the Russian Ministry of Justice 03.07.2013 number 27572).

7. Fixed assets. - [Electronic resource] - Access mode: http://www.gks.ru/wps/wcm/connect/rosstat_main/rosstat/ru/statistics/enterpris e/fund.

8. Official statistical methodology of calculating price indices for products (costs, services) for investment purposes. Rosstat Order of 06.11.2014 number 635.

9. Pricing and budget normalization in the construction of: a tutorial / MA Queen. - 2 nd ed., Ext. and rev. - Ekaterinburg: Publishing House of the Ural Mountains. University Press, 2014. - 263p. 
УДК 658.624

Искендерова Э.Т., Исмаилов Р.Т.

\title{
ОСНОВНЫЕ ХАРАКТЕРИСТИКИ И ОСОБЕННОСТИ УПРАВЛЕНИЯ ПРОИЗВОДСТВЕННЫМ ПОТЕНЦИАЛОМ СТРОИТЕЛЬНОГО ПРЕД- ПРИЯТИЯ
}

\author{
E.T. Iskenderova, R.T.Ismailov
}

\section{MAIN FEATURES AND CHARACTERISTICS OF PRODUCTION POTEN- TIAL MANAGEMENT OF THE BUILDING ENTERPRISE}

Аннотация. Рассмотрены актуальные проблемы поиска методов эффективного управления использованием и развитием производственного потенциала строительного предприятия. Уточнено содержание основных характеристик производственного потенциала строительного предприятия. Установлено, что взаимозаменяемость элементов производственного потенциала не следует понимать чисто механически, хотя в ее основе и лежат технические и технологические особенности производства. Взаимозаменяемость элементов производственного потенциала является проблемой в большей степени экономической.

Выявлень и систематизированы характерные особенности производственного потенииала строительного предприятия. С учетом этого, предлагаются различные подходы к организации управления эффективным использованием и развитием производственного потенщиала в нестабильной экономической среде.

Ключевые слова: строительное предприятие, элементы производственного потенциала, характеристики производственного потенциала, регулирование структуры производственного потенщиала.

Abstract. The paper touches upon some topical issues of effective management of the use and development of the building enterprise production potential. The main characteristics of the production capacity of the construction enterprise are specified. It is established that the interchangeability of the elements of production potential should not be understood mechanically, although some technical and technological peculiarities of production are in its basis.

The interchangeability of the elements of production potential is an economic problem to a greater extent.

We identify and classify the typical features of the construction enterprise productive capacity. Taken in into account, different approaches to managing the effective use and development of productive capacity in an unstable economic environment are proposed. 
Key words: building enterprise, elements of the production potential, features of the production potential, structure of the production potential regulation.

Введение. Одной из актуальных проблем эффективной работы строительного предприятия (СП) является формирование и функционирование системы управления использованием и развитием его производственного потенциала. Производственный потенциал строительного предприятия фактически представляет собой систему, состоящую из различных взаимодействующих и совместно функционирующих элементов. Данные элементы выполняют различные функции в процессе производства и им присущи все черты, свойственные сложной системе.

Постановка задачи. К числу основных характеристик производственного потенциала СП можно отнести [1]: целостность, сложность, взаимозаменяемость и альтернативность элементов, взаимосвязь и взаимодействие элементов, способность к восприятию последних достижений НТП, гибкость, т.е. способность производить различные виды товарной строительной продукции, и адаптивность.

Целостность производственного потенциала означает, что функционирование всех его элементов подчинено достижению единой цели, связанной с выполнением запланированного объема работ в заданные сроки с требуемым качеством и минимальными издержками производства.

Данная цель достигается в процессе управления производственной деятельностью на основе следующих принципов: общности и единства целевого задания для производственного потенциала в целом и каждого его элемента в отдельности; общности критерия оценки эффективности функционирования и развития элементов производственного потенциала и всего потенциала в целом.

Учитывая, что в условиях рынка основная задача повышения эффективности строительного производства связана с переходом преимущественно на интенсивные способы развития, то, в первую очередь, интенсивно должен загружаться и эффективно использоваться производственный потенциал строительного предприятия. Этого можно достичь только в том случае, когда с одной и той же напряженностью и результативностью используются все его элементы, т. е. когда все элементы производственного потенциала сбалансированы и функционируют с максимально возможной отдачей. Следовательно, решение задачи эффективного использования производственного потенциала сводится к формированию напряженных производственных планов и сбалансированию вводимых в производство факторов.

Сложность производственного потенциала строительного предприятия проявляется, прежде всего, в наличии нескольких образующих его элементов, каждый из которых представляет собой отдельную часть потенциала, включающую, в свою очередь, несколько более мелких составляющих.

Например, потенциал строительного предприятия включает в себя основные фонды, которые в свою очередь, содержат строительные машины и меха- 
низмы, здания и сооружения. Строительные машины, в свою очередь, разделяются на подъемные краны, скреперы, грейдеры, бульдозеры, экскаваторы и т. д. Следовательно, производственный потенциал строительного предприятия представляет собой совокупность большого количества первичных подсистем, которые могут функционировать и развиваться как самостоятельно независимо друг от друга, так и в тесном взаимодействии между собой.

Методы исследования. Учитывая непредсказуемость «поведения» отдельных составляющих производственного потенциала в нестабильных условиях экономической среды, оценка эффективности их использования в производственном процессе носит стохастический характер, и определяется статистическими методами.

Другим признаком сложности является то, что изменения, происходящие в каком либо одном элементе производственного потенциала, требуют изменения и других его элементах. Например, внедрение в производство высокопроизводительных экскаваторов для рытья строительных котлованов высвобождает и позволяет использовать по другому назначению производственных рабочих.

Еще один признак сложности определяется наличием в системе управления производственным потенциалом и в самом производственном потенциале обратных материально-вещественных, информационных и управленческих связей. Таким образом, учитывая сложность производственного потенциала СП, для его управления необходимо использовать соответствующие принципы управления сложными объектами [2].

Важной характерной особенностью производственного потенциала является взаимозаменяемость и альтернативность его элементов. Эффективная замена одного его элемента на другой является одной из важнейших проблем оперативного управления производственным процессом.

Например, увеличение массы активной части основных фондов (количество задействованных в производстве строительных машин и механизмов) как правило, приводит к значительному снижению необходимости в таком элементе производственного потенциала, как рабочая сила. Кроме того, замена одного элемента производственного потенциала на другой сказывается на эффективности строительного производства, например, когда различные строительно-монтажные работы, выполняемые в «ручную» заменяются работами, производимыми механизированным образом. В то же время дополнительное привлечение дешевой рабочей силы при расширении производства в случае ее избытка на рынке труда в регионе, снижает в структуре производственного потенциала строительного предприятия объемы энергетического элемента и активной части основных производственных фондов. Внедрение на строительном предприятии более прогрессивных технологий строительного производства и принципов его организации также снижает потребность в других элементах производственного потенциала. 
Взаимозаменяемость элементов производственного потенциала является одной из наиболее изученных его характеристик. Особенно активно исследовались вопросы замещения живого труда активной частью основных производственных фондов. Например, экономию живого труда в рамках процесса развития активной части основных производственных фондов можно регулировать, используя соотношение А. Толкачева:

$$
\Pi=\frac{P_{1} / P_{0}-1}{S_{1}-S_{0}}
$$

где $\Pi$ - коэффициент замещения живого труда элементами основных производственных фондов; $P_{0}, P_{1}$ - производительность труда, соответственно, до и после проведенного замещения; $S_{0}, S_{1}$ - соответственно фондовооруженность труда в данные периоды.

В этом случае, устанавливая пороговое значение $\Pi^{*}$ коэффициента замещения $\Pi$, можно определить такие значения показателей $P_{1}$ и $S$, , чтобы для фактического значения коэффициент замещения живого труда элементами основных производственных фондов ПФ выполнялось условие: «П $\geq \prod^{*} \gg$.

При этом следует иметь в виду, что взаимозаменяемость элементов производственного потенциала не следует понимать чисто механически, хотя в ее основе и лежат технические и технологические особенности производства.

Взаимозаменяемость элементов производственного потенциала является проблемой в большей степени экономической. Поэтому в условиях рынка в качестве основного критерия взаимозаменяемости элементов потенциала следует рассматривать получение дополнительной прибыли путем снижения издержек производства. В этой связи перед руководством строительного предприятия возникает задача обеспечения эффективных условий взаимозаменяемости элементов производственного потенциала. Однако в этом случае необходимо учитывать то обстоятельство, что степень альтернативности элементов производственного потенциала является переменной величиной и зависит от ситуации сложившейся в экономической среде СП. Она изменяется, например, в зависимости от уровня развития производственного потенциала, а также от особенностей хозяйственной системы: масштабности, характера деятельности и степени ее замкнутости.

Решение проблемы взаимозаменяемости элементов производственного потенциала, в конечном счете, сводится к выяснению того, является ли экономически целесообразной замена одного элемента производственного потенциала на другой элемент. Если такая замена приносит дополнительную прибыль, то при каких пропорциях это происходит, каковы особенности такой замены и имеются ли для этого условия, а также каковы последствия проводимой замены для развития производственного потенциала строительного предприятия.

Обсуждение результатов. Наиболее характерными особенностями взаимозаменяемости элементов производственного потенциала являются регламентированность и периодичность. Первая особенность проявляетсяв том, что все 
элементы могут теоретически неограниченно заменять друг друга до тех пор, пока один из них не обратится в ноль.

Однако на практике для организации эффективного строительного производства необходимо присутствие всех элементов производственного потенциала. Поэтому границы взаимозаменяемости производственных ресурсов определяются реально существующими ограничениями [1].

Нижняя граница взаимозаменяемости производственных факторов одного другим при этом характеризуется таким их соотношением, которое соответствует простейшим производственным технологиям, существующим в данный момент времени, а верхняя граница характеризуется соотношением между производственными факторами, характерными для наиболее прогрессивных производственных технологий.

Обычно пределы взаимозаменяемости элементов производственного потенциала определяются внешними условиями и зависят от уровня научнотехнического развития отрасли, уровня развития рыночных отношений и состояния рынка труда. В соответствии с этим границы взаимозаменяемости элементов производственного потенциала следует определить обобщенно, например, согласно следующему критерию:

« Любой элемент может замещуаться другим пока это выгодно и не тормозит сбалансированного развития производственного потенциила».

Второе свойство взаимозаменяемости элементов определяется ее дискретностью и периодичностью, означающими, что взаимозаменяемость не является непрерывной ни по количественным характеристикам, ни во времени. Это, прежде всего, определяется такой характеристикой как срок морального износа орудий и средств труда. Наряду с этим, замена одних элементов другими часто требует финансовых средств, а для их накопления требуется время. Последнее условие в определенной степени смягчается мощностью и уровнем эффективности использования производственного потенциала. Причем, чем они выше, тем короче принимается обменный цикл.

Взаимозаменяемость элементов потенциала[1], обладает еще одной фундаментальной особенностью - способностью достигать сбалансированного равновесия элементов. Это означает, что если доля одного из элементов возрастает за счет снижения доли другого элемента, потому, что первый эффективней, то настанет такой момент, когда более эффективный элемент будет вовлекаться в хозяйственный оборот в таких масштабах, которые сделают уже неэффективным дальнейшее углубление взаимозаменяемости. Наступит момент, когда оба используемых элемента будут одинаково эффективными или неэффективными, т.е. для производственного потенциала СП достигается их равновесие.

Следовательно, можно говорить об оптимальной сбалансированности элементов производственного потенциала, т.е. когда в производстве участвуют все элементы потенциала в таких пропорциях, при которых достигается максимум или минимум, в соответствии с содержанием, функции эффективности функ- 
ционирования СП, например, сокращение сроков строительства или получение максимальной прибыли.

Таким образом, основной задачей управления производственным потенциалом СП является оптимальная его сбалансированность с учетом воздействия различных внутренних и внешних факторов экономической среды. Оптимальная сбалансированность производственного потенциала достигается путем решения следующей задачи. Необходимо найти такие пропорции между элементов производственного потенциала при заданных ресурсных ограничениях, при которых СП получает максимальную прибыль с учетом внешних и внутренних условий функционирования [3].

Наконец, к особенности взаимозаменяемости элементов производственного потенциала следует отнести такое свойство как индуктивность, которая проявляется в том, что замена одного элемента другим во многих случаях создает возможность для ряда других замещений или даже делает их необходимыми.

Четвертой характеристикой производственного потенциала СП является взаимосвязь и взаимодействие его элементов в процессе совместного функционирования. Она представляет собой общий экономический закон соответствия между основными элементами производственных сил. Иными словами, между различными факторами производства существует объективно обусловленная количественная и качественная взаимосвязь, выраженная мерой их соответствия и соотношения.

Для наглядности таких взаимосвязей и качественного сбалансирования элементов производственного потенциала удобно использовать матрицы соответствия, строки и столбцы которых определяются элементами производственного потенциала или их качественными характеристиками, а элементы матриц помечаются характером связей элементов и их качественными показателями $[4]$.

Взаимодействие между элементами потенциала в строительном производстве достигается установлением связей между ними, обеспечивающими их совместное сбалансированное использование в производственном процессе, в результате чего образуются новые качества, не присущие отдельным элементам взаимодействия. Поэтому элементы производственного потенциала СП способны взаимно дополнять друг друга, то есть их влияние на эффективность производственного процесса проявляется интегрально.

Это очень важная отличительная черта производственного потенциала СП. Она свидетельствует о том, что форма взаимосвязи элементов влияет на отдачу производственного потенциала, его динамичность и адаптивность структуры. При этом отсутствие или нарушение места или функций одного из элементов потенциал оказывает влияние на соответствующее данным изменениям свойство, качественно характеризующие их совместное функционирование.

Для управления взаимосвязью и взаимодействием различных элементов производственного потенциала, т.е. для определения управленческих мероприятий, которые необходимо провести при изменении одного из элементов, 
можно использовать информационно-аналитическую модель регулирования его структуры, состоящую из системы продукций, имеющих следующее содержание:

«Если элемент, А производственного потенциала под действием внешних и внутренних факторов развития производства претерпевает качественнье изменения $\boldsymbol{C}$, то связанный с ним элемент $\boldsymbol{B}$ отношением $\boldsymbol{D}$ должен также претерпеть качественнье изменения $\boldsymbol{C}^{*}$ при условии соблюдения оптимальной сбалансированности всех составляющих производственного потенциала в цуелом».

Необходимость такого регулирования объясняется тем, что с улучшением только какого-то одного элемента невозможно добиться существенного роста отдачи производственного потенциала в целом. Другими словами, наивысшая отдача производственного потенциала возможна только при одновременной модернизации всех его элементов.

Наличие у производственного потенциала строительного предприятия такой характеристики, как взаимосвязь и взаимодействие элементов определяет особенности методологии его исследования, заключающейся в совокупном изучении поведения его элементов, т.к. ни один из элементов потенциала не может быть исчерпывающе изучен и понят без учета его взаимодействия с другими его элементами.

Пятым характерным признаком производственного потенциала является его способность к восприятию последних достижений научно-технического прогресса. Особенностью развития производственного потенциала строительного предприятия как сложной системы заключается в том, что его мощность, гибкость внутренней структуры элементов, а также формы взаимосвязей между ними определенным образом сказываются на отдаче производственного потенциала в целом, на его способности к развитию путем непосредственного и систематического использования новых научных достижений.

Например, разработка и внедрение новой технологии идет через преодоление используемой. Поэтому исключительно важно обеспечить высокую преемственность материальных, информационных, людских и других факторов производства при внедрении новых производственных технологий.

Эта черта производственного потенциала на сегодняшний день является практически неизученной. Однако на практике доказано, что менее восприимчивые к НТП производства, как правило, являются менее эффективными, чем производства с более высокой научно-технической емкостью, т. е. способностью использовать последние достижения научно-технического прогресса.

К тому же строительное предприятие, использующее современные производственные технологии и стройматериалы, имеют более высокую конкурентоспособность и широкие перспективы для развития. Поэтому среди экономистов сложилось мнение, что чем ниже уровень прогрессивности элементов потенциала, тем он консервативнее, тем в большей мере его качественный рост осуществляется экстенсивным путем за счет нарастания «силовых усилий» обще- 
ства и завышенных издержках производства, и, напротив, при высоком организационно-техническом уровне потенциала быстрее внедряются прогрессивные виды строительства и строительной техники, современные технологии, эффективные формы организации производства, труда и управления.

Одной из важнейших проблем повышения эффективности строительного производства является эффективное управление развитием его производственного потенциала. К основной задаче такого управления в условиях рынка следует отнести повышение конкурентоспособности производственного потенциала СП. Под конкурентоспособностью производственного потенциала в рассматриваемом случае следует понимать его способность производить требуемые объемы строительной продукции в единицу времени с заданным качеством и минимальными издержками производства. Это достигается на основе изучения спроса на товарную строительную продукцию и выбора на этой основе таких ее видов, которые позволяют запланировать объемы производства, обеспечивающие интенсивную загрузку потенциальных возможностей СП и развитие его производственного потенциала на основе последних достижений НТП. С учетом данного положения под конкурентоспособностью производственного потенциала СП следует понимать его комплексную сравнительную характеристику, позволяюшую дать оценку прогрессивности и состояния всех его элементов относительно стандартов, определяемых последними достижениями НТП.

Шестой характерной особенностью производственного потенциала СП является его гибкость. Она свидетельствует о возможности быстрой перестройки строительного производства на выпуск новой товарной продукции, вплоть до индивидуального строительства каждого отдельного уникального строительного проекта. Повышенные требования к гибкости производственного потенциала, в первую очередь, определяются нестабильностью экономической среды, возрастанием колебаний объема и структуры спроса, резким ускорением темпов НТП в строительной отрасли, и как следствие, непрерывного обновления архитектуры и планировки строящихся объектов.

Вывод. Основные характеристики производственного потенциала СП можно классифицировать по следующим признакам: внутренние свойства и особенности структуры, качественные характеристики и количественные характеристики.

К структурным особенностям потенциала следует отнести целостность, сложность, взаимозаменяемость элементов, их взаимосвязь и взаимодействие. К качественным характеристикам можно отнести способность элементов потенциала к восприятию достижений НТП, гибкость, мощность и его адаптивность. Под адаптивностью производственного потенциала следует понимать его способность приспосабливаться к изменениям экономической среды.

Следовательно, основной характеристикой, которой должен обладать производственный потенциал СП в нестабильных условиях экономической 
среды, является его адаптивность, которая может быть реализована путем изменения структуры его элементов.

К количественным характеристикам можно отнести, например, показатели эффективности использования средств производства, энергетических, информационных и технологических элементов производственного потенциала СП.

В заключение следует отметить, что различные характеристики производственного потенциала СП проявляются различным образом в соответствии с внешними и внутренними условиями его функционирования. В основном они определяются следующими факторами экономической среды: темпами развития НТП, изменением спроса, состоянием рынка ресурсов и внутренними особенностями производственного потенциала как сложной динамической с истемы.

\section{Библиографический список:}

1. Авдиенко В.Н., Котов В.А. Производственный потенциал промышленного предприятия. - М.: Экономика, 1989. - 240 с.

2. Растригин Л.А.Современные принципы управления сложными объектами. -М.: Сов. радио, 1980. 232 с.

3. Мелехин В.Б., Мелехин П.В. Методика управления сбалансированным ростом строительного производства // Экономика строительства. -2009. -№3. C. 18-22.

4. Мелехин П.В. Разработка методов оптимального управления взаимодействием и развитием элементов производственного потенциала// Вестник Дагестанского государственного технического университета. Технические науки. - 2010. - № 4. (19). -С. 108-112

\section{References:}

1. V.N. Avdienko, V.A. Kotov The production potential of the industrial enterprise. - M .: Economics, 1989. - 240 p.

2. L.A.Rastrigin Modern principles of complex objects management. -M .: Sov. radio, $1980.232 \mathrm{p}$.

3. V.B Melekhin PV Methods of control balanced growth of construction production // Construction Economics. -2009. -No. 3. - pp. 18-22.

4. V.B Melekhin PV Development of optimal control methods inter-action and the development of elements of the production potential // Bulletin of the Dagestan State Technical University. Technical science. - 2010. - № 4. (19). -FROM. pp.108112. 